\title{
Limit Theorems for the Number of Parts in a Random Weighted Partition
}

\author{
Ljuben Mutafchiev \\ American University in Bulgaria \\ 2700 Blagoevgrad, Bulgaria \\ Institute of Mathematics and Informatics \\ Bulgarian Academy of Sciences \\ ljuben@aubg.bg \\ Submitted: Aug 5, 2009; Accepted: Sep 20, 2011; Published: Oct 24, 2011 \\ Mathematics Subject Classification: 05A17, 60C05, 60F05
}

\begin{abstract}
Let $c_{m, n}$ be the number of weighted partitions of the positive integer $n$ with exactly $m$ parts, $1 \leq m \leq n$. For a given sequence $b_{k}, k \geq 1$, of part type counts (weights), the bivariate generating function of the numbers $c_{m, n}$ is given by the infinite product $\prod_{k=1}^{\infty}\left(1-u z^{k}\right)^{-b_{k}}$. Let $D(s)=\sum_{k=1}^{\infty} b_{k} k^{-s}, s=\sigma+i y$, be the Dirichlet generating series of the weights $b_{k}$. In this present paper we consider the random variable $\xi_{n}$ whose distribution is given by $P\left(\xi_{n}=m\right)=c_{m, n} /\left(\sum_{m=1}^{n} c_{m, n}\right), 1 \leq$ $m \leq n$. We find an appropriate normalization for $\xi_{n}$ and show that its limiting distribution, as $n \rightarrow \infty$, depends on properties of the series $D(s)$. In particular, we identify five different limiting distributions depending on different locations of the complex half-plane in which $D(s)$ converges.
\end{abstract}

\section{Introduction and Statement of the Results}

A weighted partition of the positive integer $n$ is a multiset of size $n$ whose decomposition into a union of disjoint components (parts) satisfies the following condition: for a given sequence of non-negative numbers $\left\{b_{k}\right\}_{k \geq 1}$, a part of size $k$ appears in exactly one of $b_{k}$ possible types. For more details and examples of multisets, we refer the reader e.g. to [3; Sect. 2.1 and 2.2]. Let $c_{m, n}$ be the number of weighted partitions of $n$, which contain exactly $m$ parts $(1 \leq m \leq n)$. It is known that, for a given parametric sequence $\left\{b_{k}\right\}_{k \geq 1}$, the bivariate generating function $f(u, z)$ of the numbers $c_{m, n}$ is of Euler's type, namely,

$$
f(u, z)=1+\sum_{n \geq 1} \sum_{1 \leq m \leq n} c_{m, n} u^{m} z^{n}=\prod_{k=1}^{\infty}\left(1-u z^{k}\right)^{-b_{k}}, \quad|u| \leq 1,|z|<1
$$


(see [25; Sect. 3.14]). Let $c_{n}=\sum_{m=1}^{n} c_{m, n}$ be the total number of weighted partitions of $n$. We introduce the uniform probability measure $P$ on the set of weighted partitions of $n$ assuming that the probability $1 / c_{n}$ is assigned to each $n$-partition. Furthermore, let $\xi_{n}$ denote the number of parts in a random partition of $n$. With respect to the probability measure $P, \xi_{n}$ becomes a random variable, defined on the set of all weighted partitions of $n$. Its distribution is given by

$$
P\left(\xi_{n}=m\right)=\frac{c_{m, n}}{c_{n}}, \quad 1 \leq m \leq n .
$$

If we let

$$
\varphi_{n}(u)=\sum_{m=1}^{n} P\left(\xi_{n}=m\right) u^{m}, \quad|u| \leq 1,
$$

to denote the probability generating function of $\xi_{n}$, then (1.1) can be written as

$$
f(u, z)=1+\sum_{n=1}^{\infty} c_{n} \varphi_{n}(u) z^{n}=\prod_{k=1}^{\infty}\left(1-u z^{k}\right)^{-b_{k}} .
$$

Setting $u=1$, we obtain the generating function of the numbers $c_{n}$ :

$$
f(1, z)=1+\sum_{n=1}^{\infty} c_{n} z^{n}=\prod_{k=1}^{\infty}\left(1-z^{k}\right)^{-b_{k}} .
$$

Many authors have investigated the asymptotic behavior of the Taylor coefficients of $f(1, z)$ in several particular cases. A fairly general scheme of assumptions on the parametric sequence $\left\{b_{k}\right\}_{k \geq 1}$ was proposed by Meinardus [16], who determined the asymptotic of the numbers $c_{n}$ as $n \rightarrow \infty$. His approach is based on considering two generating series:

$$
D(s)=\sum_{k=1}^{\infty} b_{k} k^{-s}, \quad s=\sigma+i y
$$

and

$$
G(z)=\sum_{k=1}^{\infty} b_{k} z^{k}, \quad|z| \leq 1 .
$$

Below we give Meinardus' scheme of conditions. Throughout the paper by $\Re(z)$ and $\Im(z)$ we denote the real and imaginary part of the complex number $z$, respectively.

(M1) The Dirichlet series (1.5) converges in the half-plane $\sigma>r>0$ and there is a constant $C_{0} \in(0,1)$, such that the function $D(s)$ has an analytic continuation to the half-plane $\left\{s: \sigma \geq-C_{0}\right\}$ on which it is analytic except for the simple pole at $s=r$ with residue $A>0$.

(M2) There exists a constant $C_{1}>0$ such that

$$
D(s)=O\left(|y|^{C_{1}}\right), \quad|y| \rightarrow \infty
$$


uniformly for $\sigma \geq-C_{0}$.

(M3) There are constants $C_{2}>0$ and $\nu>0$, such that the function $g(x)=G\left(e^{-x}\right), x=$ $\delta+2 \pi i \alpha, \alpha$ real and $\delta>0($ see $(1.6))$ satisfies

$$
\Re(g(x))-g(\delta) \leq-C_{2} \delta^{-\nu}, \quad|\arg (x)|>\pi / 4, \quad 0 \neq|\alpha| \leq 1 / 2,
$$

for enough small values of $\delta$.

The first condition specifies the domain $\mathcal{H}$ in which $D(s)$ has an analytic continuation. The second is related to the asymptotic behavior of $D(s)$ whenever $|\Im(s)| \rightarrow \infty$, under a fixed $\sigma \geq-C_{0}$. Functions, which are bounded by $O\left(|\Im(s)|^{c}\right), 0<c<\infty$, in certain domain, as $|\Im(s)| \rightarrow \infty$, are called functions of finite order. It is known that the sum of the Dirichlet series in (1.5) satisfies the finite order property in its half-plane of convergence (see e.g. [21; Sect. 9.4]). Meinardus' second condition requires that the same holds for the analytic continuation of $D(s)$ in the domain $\mathcal{H}$. Finally, Meinardus' third condition implies a bound on $\Re\left(G\left(e^{-x}\right)\right.$ ) (see (1.6)) for certain specific complex values of $x$. In some cases its verification is technically complicated. Recently, Granovsky et al. [7] weakened it and obtained Meinardus' theorem for the asymptotic of the numbers $c_{n}$ under a more general condition, which we state below.

(MGSE) For enough small $\delta>0$ and any $\mu>0$,

$$
\sum_{k=1}^{\infty} b_{k} e^{-k \delta} \sin ^{2}(\pi k \alpha) \geq\left(1+\frac{r}{2}+\mu\right) \frac{2}{\log 5}|\log \delta|,
$$

where $\sqrt{\delta} \leq \alpha \leq 1 / 2$.

Throughout the paper we assume that conditions (M1), (M2) and (MGSE) (or (M3)) are satisfied. Our aim is to show that the number of parts $\xi_{n}$ in a random weighted partition, appropriately normalized, converges in distribution as $n \rightarrow \infty$. We obtain five different limiting distributions depending on values of the parameter $r$ (see condition (M1)). Furthermore, recalling the exact distribution (1.2) of $\xi_{n}$, we point out that our results may be also interpreted in terms of the asymptotic of the combinatorial numbers $c_{m, n}$. The range of values of $m$ is specified by the corresponding scale factor and change of the origin in the weak convergence of $\xi_{n}$ to a non-degenerate probability distribution. Our study is also motivated by several old and recent results in this direction, obtained for particular sequences of weights $\left\{b_{k}\right\}_{k \geq 1}$ satisfying Meinardus' conditions with a specific value of the parameter $r$ (see condition (M1)). Below we give a brief review, which includes important results on the limiting behavior of the random variable $\xi_{n}$.

Consider first the simplest case of the uniform parametric sequence $b_{k}=1, k \geq 1$. For the Dirichlet series (1.5), we have $D(s)=\zeta(s)$. (Throughout the paper $\zeta(s)=$ $\sum_{k=1}^{\infty} k^{-s}, s=\sigma+i y$, will denote the Riemann zeta function.) It follows that condition (M1) holds with $r=1$ (see e.g. [24; Sect. 13.13]). In this case we encounter the socalled linear integer partitions (for more details, various properties and combinatorial interpretations, see [2] and [25; Sect. 3.14-3.16]). Erdős and Lehner [6] were apparently the first who have studied integer partitions using a probabilistic approach. As a matter of fact, they found an appropriate normalization for $\xi_{n}$ in this case and showed that 
$\pi \xi_{n} /(6 n)^{1 / 2}-\log \left((6 n)^{1 / 2} / \pi\right)$ converges weakly, as $n \rightarrow \infty$, to a random variable having the doubly exponential (extreme value) probability distribution. A local version of their theorem was derived later by Auluck et al. [4].

The next classical result in this area was obtained by Haselgrove and Temperley [9]. They found the asymptotic behavior of the numbers $c_{m, n}$ in a particular range of values of $m$ under certain set of conditions on the pairs $\left(k, b_{k}\right), k \geq 1$, and established convergence to a non-Gaussian limiting distribution for $\xi_{n}$. One of their conditions implies that Meinardus' parameter $r$ is $<2$. Haselgrove and Temperley's results were extended in some respects by Richmond [19] under the restriction $r<2$. Lee [14] introduced a proper scale factor for $\xi_{n}$ and obtained a limiting distribution under Meinardus' scheme of conditions whenever $r<1$.

Furthermore, Haselgrove and Temperley [9, p. 237] claimed that Gaussian law would appear as a limiting distribution for the number of parts in a random weighted partition of $n$ if $r \geq 2$, however, a formal proof was not given in their paper. This claim was confirmed in [12], where the particular case $b_{k}=k, k \geq 1$, was considered. Under this setting, $D(s)=\zeta(s-1)$ and therefore $r=2$. This case has important combinatorial interpretation. It turns out that the generating function $f(1, z)$ (see (1.4)) enumerates the plane partitions of $n$ and $\xi_{n}$ equals the sum of the diagonal parts (the trace) of the twodimensional array that represents $n$ as a double sum of non-negative integers. (For more details on various properties of plane partitions and their applications to combinatorics and analysis of algorithms, we refer the reader to [2, Chap. 11], [18; Chap. 11] and [20; Chap. 7].)

The problem on the asymptotic of the number of components of the other two types of decomposable structures - assemblies and selections - was studied in detail by Hwang [11].

Weighted partitions are also associated with the generalized Bose-Einstein model of ideal gas, where the parameters $b_{k}$ are interpreted as counts of the different types of particles at energy level $k, k=1,2, \ldots$; see [22]. One important particular case is whenever $b_{k}=\rho k^{r-1}, \rho>0, k \geq 1$. Granovsky et al. [7] showed that this sequence of parameters satisfies Meinardus' conditions (M1)-(M3). Vershik and Yakubovich [23] studied the asymptotic behavior of a general family of probability measures on the set of linear integer partitions of $n$. Their model includes the above parametric sequence. These probability measures are appropriately defined using the infinite product (1.4). The main goal of their paper is the study of the limiting distribution of the maximum particle energy of a system whose total energy grows. It was shown that the doubly exponential distribution appears as a limiting one for this model. As a particular case, Erdős and Lehner's result [6] was also obtained.

Before stating our main results, for the sake of brevity, we introduce the following notation:

$$
L_{n}(r)=\left(\frac{n}{A \Gamma(r+1) \zeta(r+1)}\right)^{\frac{1}{r+1}}, \quad n \geq 1,
$$

where the constants $r$ and $A$ are those defined by condition (M1). 
Theorem 1 Suppose that the parameters $b_{k}, k \geq 1$, satisfy conditions (M1), (M2) and (MGSE) (or (M3)). Then, the limiting distributions of the random variable $\xi_{n}$, as $n \rightarrow \infty$, in the whole range of values of $r$ are given in parts (i) - (v) below as follows.

(i) If $r<1$, then $\xi_{n} / L_{n}(r)$ converges in distribution to a random variable $\xi$ with moment generating function

$$
E\left(e^{t \xi}\right)=g_{1}(t)=e^{D(1) t} \prod_{k=1}^{\infty}\left(1-\frac{t}{k}\right)^{-b_{k}} e^{-b_{k} t / k}, \quad t \in(-1,1),
$$

where $D(1)$ denotes the value of the Dirichlet series (1.5) at $s=1$.

(ii) If $r=1$, then $\xi_{n} / L_{n}(1)-A \log L_{n}(1)$ converges in distribution to a random variable $\xi$ with moment generating function

$$
E\left(e^{t \xi}\right)=g_{2}(t)=e^{C t} \prod_{k=1}^{\infty}\left(1-\frac{t}{k}\right)^{-b_{k}} e^{-b_{k} t / k}, \quad t \in(-1,1),
$$

where the constant $C$ is determined by condition (M1) as follows:

$$
C=D(s+1)-\left.\frac{A}{s}\right|_{s=0} .
$$

(iii) If $1<r<2$, then $\xi_{n} / L_{n}(r)-A \Gamma(r) \zeta(r) L_{n}^{r-1}(r)$ converges in distribution to a random variable $\xi$ with moment generating function $g_{1}(t)$ given by (1.8).

(iv) If $r=2$, then

$$
\lim _{n \rightarrow \infty} P\left(\frac{\xi_{n}-\mu_{n}}{\sigma_{n}} \leq x\right)=\frac{1}{\sqrt{2 \pi}} \int_{-\infty}^{x} e^{-y^{2} / 2} d y, \quad-\infty<x<\infty,
$$

where $\mu_{n}$ and $\sigma_{n}$ are given by

$$
\mu_{n}=A \zeta(2) L_{n}^{2}(2), \quad \sigma_{n}=L_{n}(2)\left(\frac{A \log n}{3}\right)^{1 / 2} .
$$

(v) If $r>2$, then

$$
\lim _{n \rightarrow \infty} P\left(\frac{\xi_{n}-\mu_{n}^{\prime}}{\sigma_{n}^{\prime}} \leq x\right)=\frac{1}{\sqrt{2 \pi}} \int_{-\infty}^{x} e^{-y^{2} / 2} d y, \quad-\infty<x<\infty
$$

where

$$
\begin{aligned}
\mu_{n}^{\prime} & =A \Gamma(r) \zeta(r) L_{n}^{r}(r), \\
\sigma_{n}^{\prime} & =\left(A \Gamma(r)\left(\zeta(r-1)-\frac{(r-1)^{2} \zeta^{2}(r)}{r(r+1) \zeta(r+1)}\right) L_{n}^{r}(r)\right)^{1 / 2}
\end{aligned}
$$


Remark 1. Parts (iv) and (v) of our theorem prove Haselgrove and Temperley's claim [9; p. 237] about the asymptotic normality of $\xi_{n}$ whenever $r \geq 2$.

Remark 2. If $r<2$, the limiting distribution of $\xi_{n} / L_{n}(r)$ is non-Gaussian. It coincides with the limiting distributions obtained in $[9,19,14]$.

Remark 3. Let $\mathcal{A}=\left\{a_{k}, k \geq 1\right\}, 0<a_{1} \leq a_{2} \leq \ldots$, denote a sequence of positive integers satisfying

$$
\sum_{k=1}^{\infty} \frac{1}{a_{k}^{2}}<\infty
$$

Consider the class of entire functions $K(t)$ of the complex variable $t$, defined by

$$
K(t)=e^{-b t} \prod_{k=1}^{\infty}\left(1-\frac{t}{a_{k}}\right) e^{t / a_{k}}
$$

where $b$ is given real constant. The functions $K(t)$ (without any restriction on the reals $a_{k}, k \geq 1$ ) have been studied long ago by Laguerre, who has shown that representation (1.16) uniquely describes the class of functions, which are uniform limits of polynomials with only real roots. A comprehensive description of this theory is given in the book of Hirschman and Widder [10]. To obtain an alternative expression for $K(t)$ and describe its relationship with parts (i)-(iii) of the theorem, we notice that the positive integers $a_{k}$ of the above sequence $\mathcal{A}$ may repeat their values. So, for any positive integer $k$, we let $b_{k}$ to denote the number of those $a_{j}$ 's which are $=k$. Then, we can rewrite (1.16) as follows:

$$
K(t)=e^{-b t} \prod_{k=1}^{\infty}\left(1-\frac{t}{k}\right)^{b_{k}} e^{b_{k} t / k} .
$$

Furthermore, if $D(s)$ denotes the Dirichlet generating series (1.5) of the sequence $b_{k}, k \geq 1$, then (1.15) implies that

$$
\sum_{k=1}^{\infty} \frac{1}{a_{k}^{2}}=\sum_{k=1}^{\infty} \frac{b_{k}}{k^{2}}=D(2)<\infty .
$$

Therefore, the parameter $r$ from condition (M1) is $<2$. Consider now the function $1 / K(t)$. It is meromorphic and has poles at $t=a_{k}, k \geq 1$. Hirschman and Widder [10; Chap. III, Sect. 6] showed that if $K(t)$ satisfies (1.15) and is different from $e^{-b t} S(t)$, where $S(t)$ is a polynomial, then the inverse

$$
F(x)=\frac{1}{2 \pi i} \int_{-i \infty}^{i \infty} \frac{e^{x t}}{K(t)} d t, \quad-\infty<x<\infty,
$$

is a probability density function, which is infinitely many times differentiable and has mean $-b$ and variance $\sum_{k=1}^{\infty} 1 / a_{k}^{2}=D(2)$. Recalling (1.8), (1.9) and (1.17), we see that the moment generating functions $g_{j}(t), j=1,2$, are functions of the type:

$$
\frac{1}{K(t)}=e^{b t} \prod_{k=1}^{\infty}\left(1-\frac{t}{k}\right)^{-b_{k}} e^{-b_{k} t / k},
$$


i.e. their inverses (the probability density functions of the random variable $\xi$ ) can be computed using formula (1.18). Moreover, $b=D(1)$ in cases (i) and (iii) (see eq. (1.8)) and $b=C$ in case (ii) (see eq.(1.9)). It seems that Haselgrove and Temperley were the first who applied functions of the type $1 / K(t)$ to problems related to integer partitions.

Remark 4. The remark above and other combinatorial problems (see e.g. the "money changing problem" discussed in detail in [25; Sect. 3.15]) imply that one has to assume that $b_{k}, k \geq 1$, are non-negative integers. On the other hand, it turns out that this requirement is not necessary for the analytical approach used in this paper. That is why, further on we shall consider $b_{k}, k \geq 1$, as a sequence of real non-negative numbers.

The method of our proof is based on a probabilistic representation for the Taylor coefficients in (1.3) and (1.4). It is due to Khintchine [13] who applied it in a thermodynamics context. We also use complex integration and rely our results on the asymptotic representation of the numbers $c_{n}$, presented in [7]. We choose the free parameter (denoted further by $\delta$ ) that locates the center of Khintchine's auxiliary probability distribution in the same way as in [7]. The subsequent passage to the limit, as $n \rightarrow \infty$, is based on a recent modification of Curtiss moment generating function continuity theorem [5], established by Mukherjea et al. [17]. This modification shows that the convergence in distribution holds if the corresponding sequence of moment generating functions converges pointwise in an open interval not necessarily containing the origin.

We organize our paper as follows. Section 2 includes Khinthine's probabilistic formula and some auxiliary results, necessary for its further asymptotic analysis. Some proofs are omitted since they are given in [7]. The proof of Theorem 1 is completed in Section 3, where complex analysis is applied to obtain the limiting distributions in each of the cases (i)-(v). Section 4 contains three important combinatorial examples. We show how our theorem applies to linear and plane partitions of $n$ and to the generalized Bose-Einstein model of ideal gas $[22,23]$.

\section{Preliminary Results}

First, we introduce the sequence of truncated generating functions $f_{n}$ :

$$
f_{n}(u, z)=\prod_{k=1}^{n}\left(1-u z^{k}\right)^{-b_{k}}=\sum_{1 \leq k \leq l \leq n} c_{k, l} u^{k} z^{l}, \quad|u| \leq 1,|z|<1, n \geq 1,
$$

corresponding to the right-hand sides of (1.1) and (1.3). Setting $z=e^{-\delta+2 \pi i \alpha}, \alpha \in \mathbf{R}$, where $\delta>0$ is a free parameter and applying Cauchy coefficient formula, we obtain

$$
\begin{aligned}
& c_{n} \varphi_{n}(u)=e^{\delta n} \int_{0}^{1} f\left(u, e^{-\delta+2 \pi i \alpha}\right) e^{-2 \pi i \alpha n} d \alpha \\
& =e^{\delta n} \int_{0}^{1}\left(\prod_{k=1}^{n}\left(1-u e^{-\delta k+2 \pi i \alpha k}\right)^{-b_{k}}\right) e^{-2 \pi i \alpha n} d \alpha \\
& =e^{\delta n} \int_{0}^{1} f_{n}\left(u, e^{-\delta+2 \pi i \alpha}\right) e^{-2 \pi i \alpha n} d \alpha .
\end{aligned}
$$


The last two equalities follow from the orthogonality property of the functions $e^{-2 \pi i \alpha n}, n \geq$ 1. On the other hand, for $u \in(0,1]$, we have the binomial expansion

$$
\left(1-u z^{k}\right)^{-b_{k}}=\sum_{j=0}^{\infty}\left(\begin{array}{c}
b_{k}+j-1 \\
j
\end{array}\right) u^{j} z^{k j} .
$$

Based on it, we introduce the sequence of independent and integer valued random variables $X_{k}=X_{k}(u, \delta), k \geq 1$, depending on $b_{k}, u$ and $\delta$, in the following way:

$$
P\left(X_{k}=j k\right)=\left(\begin{array}{c}
b_{k}+j-1 \\
j
\end{array}\right) u^{j} e^{-\delta k j}\left(1-u e^{-\delta k}\right)^{b_{k}}, \quad j \geq 0,0<u \leq 1 .
$$

Hence

$$
E\left(e^{2 \pi i \alpha X_{k}(u, \delta)}\right)=\left(\frac{1-u e^{-\delta k}}{1-u e^{-\delta k+2 \pi i \alpha k}}\right)^{b_{k}}, \quad k \geq 1 .
$$

Furthermore, let

$$
Z_{n}=Z_{n}(u, \delta)=\sum_{k=1}^{n} X_{k}(u, \delta), \quad n \geq 1
$$

By the independence assumption on $X_{k}$ and (2.1),

$$
\begin{aligned}
& \psi_{n}(\alpha, u):=E\left(e^{2 \pi i \alpha Z_{n}(u, \delta)}\right) \\
& =\prod_{k=1}^{n}\left(\frac{1-u e^{-\delta k}}{1-u e^{-\delta k+2 \pi i \alpha k}}\right)^{b_{k}}=\frac{f_{n}\left(u, e^{-\delta+2 \pi i \alpha}\right)}{f_{n}\left(u, e^{-\delta}\right)}, \quad n \geq 1 .
\end{aligned}
$$

Therefore, using the inversion formula for characteristic functions [15; Sect.3.2] and (2.2), we obtain

$$
c_{n} \varphi_{n}(u)=e^{n \delta} f_{n}\left(u, e^{-\delta}\right) P\left(Z_{n}(u, \delta)=n\right), \quad n \geq 1 .
$$

Setting here $u=1$, we arrive at the representation for the numbers $c_{n}, n \geq 1$, given in [7; formula (31)]:

$$
c_{n}=e^{n \delta} f_{n}\left(1, e^{-\delta}\right) P\left(Z_{n}(1, \delta)=n\right) .
$$

Hence, for any value of the parameter $\delta>0$,

$$
\varphi_{n}(u)=\frac{f_{n}\left(u, e^{-\delta}\right)}{f_{n}\left(1, e^{-\delta}\right)} \frac{P\left(Z_{n}(u, \delta)=n\right)}{P\left(Z_{n}(1, \delta)=n\right)}, \quad n \geq 1 .
$$

Next, we propose the choice of the parameter $\delta$. We take $\delta=\delta_{n}$ to be the solution of the equation

$$
E\left(Z_{n}(1, \delta)\right)=-\left(\log f_{n}\left(1, e^{-\delta}\right)\right)^{\prime}=\sum_{k=1}^{n} \frac{k b_{k} e^{-k \delta_{n}}}{1-e^{-k \delta_{n}}}=n, \quad n \geq 1
$$

(recall (2.3) - (2.5)). In thermodynamics this quantity has the meaning of the entropy of the system. This important fact that clarifies the choice of the free parameter was 
observed by Khintchine [13; Chap. VI]; see also [7] and the references therein for further developments of the method.

The asymptotic form of the unique solution of (2.7) was determined by Granovsky et al. [7; formula (43)]. We have

$$
\delta=\delta_{n}=L_{n}^{-1}(r)+\frac{D(0)}{r+1} n^{-1}+O\left(n^{-1-\beta}\right),
$$

where $L_{n}(r)$ is the quantity given by $(1.7)$ and

$$
\beta=\left\{\begin{array}{lll}
\frac{C_{0}}{r+1} & \text { if } & C_{0} \geq r, \\
\frac{r}{r+1} & \text { if } & C_{0}<r .
\end{array}\right.
$$

Further, we set $u=e^{-w \delta}$ and $\mathcal{F}(w, \delta)=f\left(e^{-w \delta}, e^{-\delta}\right), w \geq 0$. In view of (1.1), we have

$$
\mathcal{F}(w, \delta)=\prod_{k=1}^{\infty}\left(1-e^{-\delta(k+w)}\right)^{-b_{k}}
$$

$\mathcal{F}(w, \delta)$ admits an integral representation, similar to that established in $[16,7]$ for $\mathcal{F}(0, \delta)$. Namely, for any $\delta>0$ and $w \geq 0$, we have

$$
\log \mathcal{F}(w, \delta)=\frac{1}{2 \pi i} \int_{1+r-i \infty}^{1+r+i \infty} \delta^{-s} \Gamma(s) D(s, w) \zeta(s+1) d s,
$$

where $D(s, w)$ is the shifted Dirichlet series

$$
D(s, w)=\sum_{k=1}^{\infty} b_{k}(k+w)^{-s}, \quad s=\sigma+i y,
$$

and $D(s, 0)=D(s)$ (see $(1.5)$ ). In the next lemma we establish a series expansion of $D(s, w)$ in powers of $w$ and show that it is valid for all $|w|<1$. It allows the computation of the integral in (2.10) via residue theorem. In what follows we denote by $\{r\}$ the fractional part of $r$.

Lemma 1 Suppose that the sequence $b_{k}, k \geq 1$, is such that the associated Dirichlet series $D(s)$ satisfies conditions (M1) and (M2). Then,

(i) for fixed s with $\sigma \geq-C_{0}$, the shifted Dirichlet series (2.11) has the following Taylor series expansion in $w$ :

$$
D(s, w)=D(s)+\sum_{l=1}^{\infty} \frac{(-w)^{l}}{l !} \frac{\Gamma(s+l)}{\Gamma(s)} D(s+l), \quad s=\sigma+i y ;
$$

(ii) the function $D(s, w)$ is meromorphic in $s$ for $\sigma \geq-C_{0}, C_{0} \in(0,1)$, and has only simple poles at $s=r, r-1, \ldots, d(r)$, where

$$
d(r)=\left\{\begin{array}{lll}
1 & \text { if } & r \text { is an integer, } \\
\{r\} & \text { if } & \{r\}>0 \text { and }\{r\}-1 \leq-C_{0}, \\
\{r\}-1 & \text { if } \quad\{r\}>0 \text { and }\{r\}-1>-C_{0}
\end{array}\right.
$$

(iii) the series in the right-hand side of (2.12) converges absolutely for $|w|<1$ if $\sigma \geq-C_{0}$ and $s \neq r, r-1, \ldots, d(r)$. 
Proof. (i) For $\sigma>r$, differentiating the series (2.11) with respect to $w$, we obtain

$$
\begin{aligned}
\left.\frac{\partial^{l}}{\partial w^{l}} D(s, w)\right|_{w=0} & =(-s)(-s-1) \ldots(-s-l+1) D(s+l, 0) \\
& =(-1)^{l} s(s+1) \ldots(s+l-1) D(s+l, 0), \quad l=1,2, \ldots
\end{aligned}
$$

which yields the Taylor series expansion in (2.12). The analytical continuation of $D(s+$ $l, 0)=D(s+l)$, stated in condition (M1), implies that (2.12) holds for $\sigma \geq-C_{0}$ and proves part (ii) of the lemma. Finally, the absolute convergence, stated in part (iii), follows immediately from the ratio test, Stirling's formula for the gamma function [24; Sect.12.33] and the fact that $|D(s+l)| \rightarrow b_{1}$ as $l \rightarrow \infty$, for any fixed $s$ satisfying the conditions of (iii).

Remark. Consider again the parametric sequence $b_{k}=1, k \geq 1$, which leads to the enumeration of the linear integer partitions of $n$ (see (1.4)). It is clear that (2.11) implies that

$$
D(s, w)=\sum_{k=0}^{\infty}(k+1+w)^{-s}=\zeta(s, 1+w),
$$

where $\zeta(s, x)$ denotes the Hurwitz zeta function [24; Sect. 13.11]. From the Taylor series expansion (2.12) it follows that

$$
D(0, w)=\zeta(0,1+w)=\zeta(0)+\left.(-w) s \zeta(s+1)\right|_{s=0}=-\frac{1}{2}-w,
$$

since in this case $D(s)=\zeta(s)$ and $\zeta(0)=-1 / 2$ [24; Sect. 13.14]. By (2.14) we have $\zeta(s, w)=\zeta(s, 1+w)+w^{-s}$, and thus we obtain the known formula for the Hurwitz zeta function

$$
\zeta(0, w)=-\frac{1}{2}-w+1=\frac{1}{2}-w
$$

(see [24; Sect. 13.21]). In a similar way, one can also recover the formulas for $\zeta(-k, w), k=$ $1,2, \ldots$, given in [24; Sect. 13.14].

Our further analysis of the asymptotic behavior of the generating function $\varphi_{n}\left(e^{-w \delta_{n}}\right)$ stems from eq. (2.6). To study the asymptotic behavior of the second factor in its righthand side we need to introduce the following notations for the semi-invariants of the random variable $Z_{n}(u, \delta)$ :

$$
\begin{gathered}
M_{n}(u)=E\left(Z_{n}(u, \delta)\right)=\left.\frac{1}{2 \pi i} \frac{\partial}{\partial \alpha} \log \psi_{n}(\alpha, u)\right|_{\alpha=0} \\
=-\frac{\partial}{\partial \delta} \log f_{n}\left(u, e^{-\delta}\right)=\sum_{k=1}^{n} \frac{u k b_{k} e^{-\delta k}}{1-u e^{-\delta k}}, \\
B_{n}^{2}(u)=\operatorname{Var}\left(Z_{n}(u, \delta)\right)=\left.\frac{1}{(2 \pi i)^{2}} \frac{\partial^{2}}{\partial \alpha^{2}} \log \psi_{n}(\alpha, u)\right|_{\alpha=0} \\
=\frac{\partial^{2}}{\partial \delta^{2}} \log f_{n}\left(u, e^{-\delta}\right)=\sum_{k=1}^{n} u k^{2} b_{k} \frac{e^{-\delta k}}{\left(1-u e^{-\delta k}\right)^{2}},
\end{gathered}
$$




$$
\begin{aligned}
& T_{n}(u)=\left.\frac{1}{(2 \pi i)^{3}} \frac{\partial^{3}}{\partial \alpha^{3}} \log \psi_{n}(\alpha, u)\right|_{\alpha=0} \\
& =-\frac{\partial^{3}}{\partial \delta^{3}} \log f_{n}\left(u, e^{-\delta}\right)=\sum_{k=1}^{n} u k^{3} b_{k} \frac{e^{-\delta k}\left(1+u e^{-\delta k}\right)}{\left(1-u e^{-\delta_{n} k}\right)^{3}} .
\end{aligned}
$$

(For the definition of the semi-invariants, see [15; Sect. 2.4].) The following lemma shows how $\mathcal{F}(w, \delta)$ (see $(2.9)$ and $(2.10)$ ) and its logarithmic derivatives with respect to $\delta$ will be used in our further computations.

Lemma 2 Suppose that the sequence $b_{k} \geq 0, k \geq 1$, is such that the associated Dirichlet series $D(s)$, defined by (1.5), satisfies the conditions of Lemma 1. If $\delta_{n}$ is the solution of (2.7) given by (2.8), then

$$
\begin{gathered}
\log f_{n}\left(e^{-w \delta_{n}}, e^{-\delta_{n}}\right)=\log \mathcal{F}\left(w, \delta_{n}\right)+\Omega_{n, 1}\left(\delta_{n}, w\right), \\
M_{n}\left(e^{-w \delta_{n}}\right)=-\frac{\partial}{\partial \delta_{n}} \log \mathcal{F}\left(w, \delta_{n}\right)+\Omega_{n, 2}\left(\delta_{n}, w\right), \\
B_{n}^{2}\left(e^{-w \delta_{n}}\right)=\frac{\partial^{2}}{\partial \delta_{n}^{2}} \log \mathcal{F}\left(w, \delta_{n}\right)+\Omega_{n, 3}\left(\delta_{n}, w\right), \\
T_{n}\left(e^{-w \delta_{n}}\right)=-\frac{\partial^{3}}{\partial \delta_{n}^{3}} \log \mathcal{F}\left(w, \delta_{n}\right)+\Omega_{n, 4}\left(\delta_{n}, w\right),
\end{gathered}
$$

where $\Omega_{n, j}\left(\delta_{n}, w\right)=o\left(\delta_{n}^{-j} n^{r+j-1} e^{-n \delta_{n}}\right)=o(1), j=1,2,3,4$, as $n \rightarrow \infty$ uniformly for $0 \leq w<1$.

Proof. From (2.1) and (2.9) it follows that

$$
\log f_{n}\left(e^{-w \delta_{n}}, e^{-\delta_{n}}\right)=\log \mathcal{F}\left(w, \delta_{n}\right)+\Omega_{n, 1}\left(\delta_{n}, w\right),
$$

where

$$
\Omega_{n, 1}\left(\delta_{n}, w\right)=\sum_{k \geq n+1} b_{k} \log \left(1-e^{-(k+w) \delta_{n}}\right) .
$$

To estimate the error term in (2.22), we use the inequality $|\log (1-x)| \leq \frac{x}{1-x}$ for $0 \leq x<$ 1 and the fact that $\frac{x}{1-x}$ is an increasing function. Thus, for any $w \in[0,1)$ and sufficiently large $n$, we observe that

$$
\begin{aligned}
\left|\Omega_{n, 1}\left(\delta_{n}, w\right)\right| & \leq \sum_{k \geq n+1} b_{k} \frac{e^{-(k+w) \delta_{n}}}{1-e^{-(k+w) \delta_{n}}} \\
& \leq \sum_{k \geq n+1} b_{k} \frac{e^{-k \delta_{n}}}{1-e^{-k \delta_{n}}}=O\left(\sum_{k \geq n+1} b_{k} e^{-k \delta_{n}}\right),
\end{aligned}
$$

since (2.8) and (1.7) imply that $n \delta_{n} \rightarrow \infty$ as $n \rightarrow \infty$. Further on, we base our argument upon a bound on the rate of growth of the parameters $b_{k}$, as $k \rightarrow \infty$. Granovsky et al. 
[7; p. 309] (see also the references therein) showed that conditions (M1), (M2) and a Tauberian theorem due to Wiener-Ikehara imply that $b_{k}=o\left(k^{r}\right)$ as $k \rightarrow \infty$. Hence we have

$$
\begin{aligned}
& \sum_{k \geq n+1} b_{k} e^{-k \delta_{n}}=o\left(\sum_{k \geq n+1} k^{r} e^{-k \delta_{n}}\right)=o\left(\int_{n+1}^{\infty} x^{r} e^{-x \delta_{n}} d x\right) \\
& =o\left(\delta_{n}^{-r-1} \int_{n \delta_{n}}^{\infty} y^{r} e^{-y} d y\right)=o\left(\delta_{n}^{-1} n^{r} e^{-n \delta_{n}}\right), \quad n \rightarrow \infty,
\end{aligned}
$$

where the last estimate follows immediately from L'Hopital's rule. Furthermore (2.8) and (1.7) yield $\delta_{n}^{-1} n^{r} e^{-n \delta_{n}}=o(1)$. Combining this with (2.22) and (2.23), we obtain (2.18) and the $o$-estimate for the function $\Omega_{n, 1}\left(\delta_{n}, w\right)$. The proofs of (2.19)-(2.21) are similar.

With the aid of (2.19)-(2.21) and complex integration one can find the asymptotic behavior of the semi-invariants (2.15)-(2.17) if $u=e^{-w \delta_{n}}$ and $n \rightarrow \infty$. The argument is based on a computation of the residuals of the integrand in (2.10), demonstrated in Section 3. Therefore, in the proof we shall refer sometimes to formulas established subsequently in Section 3.

Lemma 3 Suppose that the sequence $b_{k}, k \geq 1$, satisfies the conditions of Lemma 1 . Then, as $n \rightarrow \infty$,

$$
\begin{aligned}
& M_{n}\left(e^{-w \delta_{n}}\right)= \begin{cases}n+O\left(\delta_{n}^{-\max \{r, 1\}}\right) & \text { if } r<2, \\
n-A \zeta(2) w \delta_{n}^{-2}+O\left(\delta_{n}^{-1}\right) & \text { if } r=2, \\
n-A \Gamma(r)(r-1) \zeta(r) w \delta_{n}^{-r} & \end{cases} \\
&+O\left(w^{2} \delta_{n}^{-r+1}\right)+O\left(\delta_{n}^{-1}\right) \text { if } r>2, \\
& B_{n}^{2}\left(e^{-w \delta_{n}}\right) \sim K_{2} \delta_{n}^{-r-2}, \\
& T_{n}\left(e^{-w \delta_{n}}\right) \sim K_{3} \delta_{n}^{-r-3},
\end{aligned}
$$

uniformly for $0 \leq w<1$, where the constants $K_{j}$ are given by $K_{j}=A \Gamma(r+j) \zeta(r+1), j=$ 2,3 .

Proof. From (2.10), (3.2) and (3.3) it follows that

$$
\log \mathcal{F}\left(w, \delta_{n}\right)=H_{n}(w)+\frac{1}{2 \pi i} \int_{1+r-i \infty}^{1+r+i \infty} \delta_{n}^{-s} \Gamma(s) D(s) \zeta(s+1) d s .
$$

$H_{n}(w)$ can be evaluated using (3.11), (3.12) and (3.14). The asymptotic of the last complex integral as $\delta_{n} \rightarrow 0$ is given in [7] (see formula (39)). Substituting expression (3.12) for $H_{n}(w)$ in the right-hand side of (2.27), we obtain

$$
\begin{aligned}
\log \mathcal{F}\left(w, \delta_{n}\right)= & A \Gamma(r) \sum_{1 \leq j \leq d(r)} \delta_{n}^{-(r-j)} \zeta(r-j+1)(-w)^{j} / j !+\tilde{D}_{s}^{\prime}(0, w) \\
& -\tilde{D}(0, w) \log \delta_{n}+A \Gamma(r) \zeta(r+1) \delta_{n}^{-r}+D^{\prime}(0)-D(0) \log \delta_{n}+O\left(\delta_{n}^{C_{0}}\right) \\
= & A \Gamma(r) \sum_{1 \leq j \leq d(r)} \delta_{n}^{-(r-j)} \zeta(r-j+1)(-w)^{j} / j !+D_{s}^{\prime}(0, w) \\
& -D(0, w) \log \delta_{n}+A \Gamma(r) \zeta(r+1) \delta_{n}^{-r}+O\left(\delta_{n}^{C_{0}}\right) .
\end{aligned}
$$


As in $\left[7 ;\right.$ p. 321], we differentiate $\log \mathcal{F}\left(w, \delta_{n}\right)$ with respect to $\delta_{n}$ and observe that

$$
\begin{aligned}
-\frac{\partial}{\partial \delta_{n}} \log \mathcal{F}\left(w, \delta_{n}\right)= & A \Gamma(r) \sum_{1 \leq j \leq d(r)}(r-j) \delta_{n}^{-r-1+j} \zeta(r-j+1)(-w)^{j} / j ! \\
& +A \Gamma(r+1) \zeta(r+1) \delta_{n}^{-r-1}+D(0, w) \delta_{n}^{-1}+O\left(\delta_{n}^{C_{0}-1}\right)
\end{aligned}
$$

The asymptotic behavior of the leading term in the right-hand side of this relation can be obtained using (2.8) and (1.7). So we deduce that

$$
\begin{aligned}
& A \Gamma(r+1) \zeta(r+1) \delta_{n}^{-r-1} \\
& =n\left(1+\frac{D(0)}{(r+1) A \Gamma(r) \zeta((r+1)} n^{-\frac{r}{r+1}}+O\left(n^{-\frac{r}{r+1}-\beta}\right)\right)^{-(r+1)} \\
& =n\left(1-\frac{D(0)}{A \Gamma(r) \zeta(r+1)} n^{-\frac{r}{r+1}}+O\left(n^{-\frac{r}{r+1}-\beta}\right)\right) \\
& =n-\frac{D(0)}{A \Gamma(r) \zeta(r+1)} n^{\frac{1}{r+1}}+O\left(n^{\frac{1}{r+1}-\beta}\right)=n+O\left(\delta_{n}^{-1}\right) .
\end{aligned}
$$

Replacing this into (2.28) and using (2.19), we obtain (2.24). The asymptotic equivalences (2.25) and (2.26) follow in the same but easier way from (2.20) and (2.21), respectively. It is readily seen that the leading terms of the derivatives $\partial^{j} \log \mathcal{F}\left(w, \delta_{n}\right) / \partial \delta_{n}^{j}, j=2,3$ are equal to $K_{j} \delta_{n}^{-r-j}, j=2,3$, which completes the proof of the lemma.

Now we are ready to establish local limit theorems for the random variable $Z_{n}\left(u, \delta_{n}\right)$ whenever $u$ approaches 1 in appropriate ways. The proofs stem from (2.5). We shall use an expansion of the semi-invariant (cumulant) generating function $\log \psi_{n}(\alpha, u)$ of the random variable $Z_{n}\left(u, \delta_{n}\right)$ (see [15; Sect. 2.4]). This generating function is uniquely determined for any $u \in(0,1]$ in a neighborhood of $\alpha=0$ taking the branch of the logarithmic function for which $\log \psi_{n}(0, u)=0$. (Such a neighborhood always exists since $\psi_{n}(0, u)=1$ and $\psi_{n}(\alpha, u)$ is continuous in $\alpha$ and $\left.u \in(0,1]\right)$.

Lemma 4 Suppose that the sequence $b_{k}, k \geq 1$, satisfies conditions (M1), (M2) and (MGSE)(or (M3)). If $\delta_{n}$ is the solution of (2.7) given by (2.8), $Z_{n}(u, \delta), n \geq 1$, are the random variables defined by (2.3) and (2.4) and $B_{n}^{2}(1)$ is defined by (2.16), then, for $u=u_{n}=e^{-w \delta_{n}}$,

$$
P\left(Z_{n}\left(u_{n}, \delta_{n}\right)=n\right) \sim \frac{1}{\sqrt{2 \pi B_{n}^{2}(1)}}
$$

as $n \rightarrow \infty$, whenever the parameter $r$ from condition (M1) and the sequence $u_{n}, n \geq 1$, satisfy one of the following three conditions:

(i) $0<r<\infty$ and $u_{n}=1$;

(ii) $0<r<2$ and $u_{n}=e^{-w \delta_{n}}$, where $0<w<1$;

(iii) $r=2$ and $u_{n}=e^{-w_{n} \delta_{n}}$, where $0<w_{n}=O(1 / \sqrt{\log n})$.

Finally,

(iv) if $r>2$ and $u_{n}=e^{-w_{n} \delta_{n}}$, where

$$
0<w_{n}=w_{n}(t)=-t \delta_{n}^{\frac{r}{2}-1} /(A \Gamma(r) \zeta(r-1))^{1 / 2},
$$


and $t$ is a fixed negative real, then, as $n \rightarrow \infty$,

$$
P\left(Z_{n}\left(u_{n}, \delta_{n}\right)=n\right) \sim \frac{e^{-\lambda_{r}^{2} t^{2} / 2}}{\sqrt{2 \pi B_{n}^{2}(1)}}, \quad \lambda_{r}=\frac{(r-1) \zeta(r)}{\sqrt{r(r+1) \zeta(r-1) \zeta(r+1)}} .
$$

Proof. Part (i) of this lemma is just the local limit theorem for $Z_{n}\left(1, \delta_{n}\right)$ proved in [7; Sect. 6]. In order to prove the local limit theorem in the remaining three cases, we follow a standard argument, starting with an application of the inversion formula for characteristic functions [15; Sect. 3.2]. Next, as in [7; Sect. 6], we break up the corresponding integral into a sum of two integrals in the following way:

$$
P\left(Z_{n}\left(u_{n}, \delta_{n}\right)=n\right)=\int_{-1 / 2}^{1 / 2} \psi_{n}\left(\alpha, u_{n}\right) e^{-2 \pi i n \alpha} d \alpha=I_{1}\left(u_{n}\right)+I_{2}\left(u_{n}\right),
$$

where

$$
\begin{gathered}
I_{1}\left(u_{n}\right)=\int_{-\alpha_{0}}^{\alpha_{0}} \psi_{n}\left(\alpha, u_{n}\right) e^{-2 \pi i n \alpha} d \alpha \\
I_{2}\left(u_{n}\right)=\int_{-1 / 2}^{-\alpha_{0}} \psi_{n}\left(\alpha, u_{n}\right) e^{-2 \pi i n \alpha} d \alpha+\int_{\alpha_{0}}^{1 / 2} \psi_{n}\left(\alpha, u_{n}\right) e^{-2 \pi i n \alpha} d \alpha
\end{gathered}
$$

and

$$
\alpha_{0}=\alpha_{0}(n)=\delta_{n}^{\frac{r+2}{2}} \log ^{1 / 3} n .
$$

$I_{1}\left(u_{n}\right)$ is estimated using (2.15)-(2.17) and the expansion of the generating function of the semi-invariants of $Z_{n}\left(u_{n}, \delta_{n}\right)$ :

$$
\begin{aligned}
\psi_{n}\left(\alpha, u_{n}\right) e^{-2 \pi i n \alpha}= & \exp \left(\log \psi_{n}\left(\alpha, u_{n}\right)-2 \pi i n \alpha\right) \\
= & \exp \left(\left.\alpha\left(\frac{\partial}{\partial \alpha} \log \psi_{n}\left(\alpha, u_{n}\right)\right)\right|_{\alpha=0}+\left.\frac{\alpha^{2}}{2}\left(\frac{\partial^{2}}{\partial \alpha^{2}} \log \psi_{n}\left(\alpha, u_{n}\right)\right)\right|_{\alpha=0}\right. \\
& \left.+O\left(\left.\alpha^{3}\left(\left|\frac{\partial^{3}}{\partial \alpha^{3}} \log \psi_{n}\left(\alpha, u_{n}\right)\right|\right)\right|_{\alpha=0}\right)-2 \pi i n \alpha\right) \\
= & \exp \left(2 \pi i \alpha\left(M_{n}\left(u_{n}\right)-n\right)-2 \pi^{2} \alpha^{2} B_{n}^{2}\left(u_{n}\right)\right. \\
& \left.+O\left(\alpha^{3} T_{n}\left(u_{n}\right)\right)\right), \quad|\alpha| \leq \alpha_{0} .
\end{aligned}
$$

Granovsky et al. [7; Lemma 2] showed that

$$
B_{n}^{2}(1) \sim K_{2} \delta_{n}^{-r-2}, \quad T_{n}(1) \sim K_{3} \delta_{n}^{-r-3},
$$

where the values of the constants $K_{j}, j=2,3$, are given in the statement of Lemma 3 . We notice that the first asymptotic equivalence in (2.35) and (2.25) imply that

$$
\frac{B_{n}^{2}\left(u_{n}\right)}{B_{n}^{2}(1)} \rightarrow 1, \quad n \rightarrow \infty
$$

Then, by $(2.25),(2.26)$ and $(2.33)$,

$$
\alpha_{0} B_{n}\left(u_{n}\right) \sim K_{2}^{1 / 2} \log ^{1 / 3} n \rightarrow \infty, \quad \lim _{n \rightarrow \infty} \alpha_{0}^{3} T_{n}\left(u_{n}\right)=0 .
$$


Furthermore, (2.33), the first two equalities of (2.24) and conditions (ii) and (iii) for $u_{n}$ imply the following estimates:

$$
\alpha\left(M_{n}\left(u_{n}\right)-n\right)= \begin{cases}O\left(\delta_{n}^{r / 2} \log ^{1 / 3} n\right) & \text { if } r \leq 1 \\ O\left(\delta_{n}^{1-r / 2} \log ^{1 / 3} n\right) & \text { if } 1<r<2 \\ O\left(\log ^{-1 / 6} n\right) & \text { if } r=2\end{cases}
$$

uniformly for $|\alpha| \leq \alpha_{0}$. Therefore, the first and third term in the last exponent of (2.34) tend to 0 as $n \rightarrow \infty$ and hence the leading term in it equals $-2 \pi^{2} \alpha^{2} B_{n}\left(u_{n}\right)$. Thus, inserting this exponent into the integrand of (2.32), we get

$$
\begin{aligned}
& I_{1}\left(u_{n}\right)=\int_{-\alpha_{0}}^{\alpha_{0}} \exp \left(-2 \pi^{2} \alpha^{2} B_{n}\left(u_{n}\right)+o(1)\right) d \alpha \\
& =\frac{1}{B_{n}\left(u_{n}\right)} \int_{-\alpha_{0} B_{n}\left(u_{n}\right)}^{\alpha_{0} B_{n}\left(u_{n}\right)} \exp \left(-2 \pi^{2} z^{2}+o(1)\right) d z \\
& \sim \frac{1}{2 \pi B_{n}(1)} \int_{-\infty}^{\infty} e^{-z^{2} / 2} d z=\frac{1}{\sqrt{2 \pi B_{n}^{2}(1)}}, \quad n \rightarrow \infty .
\end{aligned}
$$

The asymptotic equivalence above follows from (2.36) and the first part of (2.37). An estimate for $I_{2}\left(u_{n}\right)$ can be obtained as in [7; Sect. 6]. The argument is essentially based on condition (MGSE). In this way one can establish that $I_{2}\left(u_{n}\right)=o\left(I_{1}\left(u_{n}\right)\right), n \rightarrow \infty$. Combining this with (2.31), (2.32) and (2.38), we complete the proof of parts (ii) and (iii) of the lemma.

To prove part (iv) we need to find the asymptotic of the first term of the exponent of (2.34). Using the last part of (2.24), under the assumption on $u_{n}$, we find that

$$
M_{n}\left(u_{n}\right)-n=\frac{A \Gamma(r)(r-1) \zeta(r) \delta_{n}^{-1-r / 2}}{(A \Gamma(r) \zeta(r-1))^{1 / 2}}+O\left(\delta_{n}^{-1}\right) .
$$

Substituting again $\alpha=z / B_{n}\left(u_{n}\right)$ in $(2.32)$, by $(2.25),(2.37)$ and the inversion formula for characteristic functions [15; Sect. 3.2], we obtain

$$
\begin{aligned}
& I_{1}\left(u_{n}\right)=\frac{1}{B_{n}\left(u_{n}\right)} \\
& \times \int_{-\alpha_{0} B_{n}\left(u_{n}\right)}^{\alpha_{0} B_{n}\left(u_{n}\right)} \exp \left(2 \pi i z t \frac{(r-1) \zeta(r)}{\sqrt{r(r+1) \zeta(r-1) \zeta(r+1)}}-2 \pi^{2} z^{2}+o(1)\right) d z \\
& \sim \frac{1}{B_{n}(1)} \int_{-\infty}^{\infty} \exp \left(2 \pi i z t \lambda_{r}-2 \pi^{2} z^{2}\right) d z \\
& =\frac{1}{2 \pi B_{n}(1)} \int_{-\infty}^{\infty} \exp \left(i y t \lambda_{r}-\frac{y^{2}}{2}\right) d y=\frac{e^{-\lambda_{r}^{2} t^{2} / 2}}{\sqrt{2 \pi B_{n}^{2}(1)}}, n \rightarrow \infty,
\end{aligned}
$$

with $\lambda_{r}$ given in (2.30). As in the previous cases, it turns out that $I_{2}\left(u_{n}\right)$ is negligible, which completes the proof of the last part of the lemma. 
Lemma 4 implies that the second factor in the right-hand side of (2.6) tends to 1 as $n \rightarrow \infty$ if $w$ and $r$ satisfy conditions (i)-(iii). Hence in these three cases

$$
\varphi_{n}\left(u_{n}\right) \sim \frac{f_{n}\left(u_{n}, e^{-\delta_{n}}\right)}{f_{n}\left(1, e^{-\delta_{n}}\right)}, \quad n \rightarrow \infty
$$

If condition (iv) of Lemma 3 holds, then an extra exponential factor appears in (2.6) and we have

$$
\varphi_{n}\left(u_{n}\right) \sim e^{-\lambda_{r}^{2} t^{2} / 2} \frac{f_{n}\left(u_{n}, e^{-\delta_{n}}\right)}{f_{n}\left(1, e^{-\delta_{n}}\right)}, \quad n \rightarrow \infty,
$$

where $\lambda_{r}$ is defined by $(2.30)$.

In the next section we show that the ratio in the right-hand side of (2.39) approaches a finite limit depending on the values of the parameter $r$.

\section{Proof of the Main Result}

We start with a brief summary on what we have observed in Section 2 .

First, with the aid of Khintchine's probabilistic representation, we obtained expression (2.6) for the generating function of the random variable $\xi_{n}$. We specified the choice $\delta=\delta_{n}$ of the parameter $\delta$ from (2.6) by eqs. (2.7) and (2.8). Then, we set $u=e^{-w \delta_{n}} \leq 1$ $(0 \leq w<1)$, introduced the function $\mathcal{F}\left(w, \delta_{n}\right)$ and found its integral representation (2.10). The last one involves the Dirichlet series $D(s, w)$ defined by (2.11). Lemma 1 establishes a series expansion in powers of $w$ of the function $D(s, w)$ that converges for $|w|<1$ (in particular, for any $w \in[0,1)$ ). It allows a computation of the residuals of the integrand in (2.10). Lemma 2 explains the role of $\mathcal{F}\left(w, \delta_{n}\right)$ in $(2.6)$ and shows how the first ratio in its right-hand side can be computed. It is also used in the computation of the semi-invariants $M_{n}\left(u_{n}\right), B_{n}^{2}\left(u_{n}\right)$ and $T_{n}\left(u_{n}\right)$. This was demonstrated in the next Lemma 3. Finally, in Lemma 4 we proved a local limit theorem for the random variable $Z_{n}\left(u_{n}, \delta_{n}\right)$. Its proof is essentially based on the results of Lemma 3. Lemma 4 allows one to study the asymptotic behavior of the second ratio in the right-hand side of (2.6).

We conclude this discussion with some comments on (2.39) and (2.40). First, we notice that if $w$ and $r$ satisfy conditions (i)-(iii) of Lemma 4, then (2.39) becomes

$$
\begin{aligned}
& \varphi_{n}\left(e^{-w \delta_{n}}\right) \sim \frac{f_{n}\left(e^{-w \delta_{n}}, e^{-\delta_{n}}\right)}{f_{n}\left(1, e^{-\delta_{n}}\right)}=\exp \left(\log \mathcal{F}\left(w, \delta_{n}\right)-\log \mathcal{F}\left(0, \delta_{n}\right)+o(1)\right) \\
& =\exp \left(H_{n}(w)+o(1)\right), \quad n \rightarrow \infty
\end{aligned}
$$

where

$$
H_{n}(w)=\frac{1}{2 \pi i} \int_{1+r-i \infty}^{1+r+i \infty} \delta_{n}^{-s} \Gamma(s) \zeta(s+1) \tilde{D}(s, w) d s
$$

and

$$
\tilde{D}(s, w)=D(s, w)-D(s)
$$


Notice that the conditions of Lemma 4(i)-(iii) imply that either $w \in[0,1)$ is fixed or $0<w=w_{n}=O(1 / \sqrt{\log n})$. Finally, if the conditions of Lemma 3(iv) are satisfied, then

$$
\begin{aligned}
& \varphi_{n}\left(\exp \left(\frac{-t \delta_{n}^{r / 2}}{(A \Gamma(r) \zeta(r-1))^{1 / 2}}\right)\right) \\
& \sim \exp \left(-\lambda_{r}^{2} t^{2} / 2+H_{n}\left(\frac{-t \delta_{n}^{r / 2-1}}{(A \Gamma(r) \zeta(r-1))^{1 / 2}}\right)\right), \\
& -1<t<0, \quad r>2, \quad n \rightarrow \infty,
\end{aligned}
$$

with $\lambda_{r}$ as in (2.30). We recall that the proofs of (3.1) and (3.4) are based on (2.10)-(2.12), (2.18) and Lemma 4 (i)-(iv).

\subsection{Computation of Residuals and Evaluation of $H_{n}(w)$}

To compute $H_{n}(w)$ we perform the integration over the contour $Q_{\tau}$ of the domain:

$$
\left\{s=\sigma+i y:-C_{0} \leq \sigma \leq r+1,|y|=\tau>0\right\} .
$$

Let

$$
J_{n}(w)=\frac{1}{2 \pi i} \int_{Q_{\tau}} \delta_{n}^{-s} \Gamma(s) \zeta(s+1) \tilde{D}(s, w) d s
$$

be the contour integral corresponding to $H_{n}(w)$. To perform the computation, we recall first the following asymptotic properties for the zeta and gamma functions:

$$
\begin{gathered}
\zeta(\sigma+1 \pm i \tau)=O\left(\tau^{C_{2}}\right), \quad \tau \rightarrow \infty, \quad C_{2}>0, \\
\Gamma(\sigma \pm i \tau)=O\left(\tau^{\sigma-1 / 2} e^{-\pi \tau / 2}\right), \quad \tau \rightarrow \infty .
\end{gathered}
$$

Eqs. (3.7) and (3.8) hold uniformly in $\sigma$ from a finite interval (see [24; Sect. 13.5] for the first one, [1, formula (6.1.45)] and [21; Sect. 4.4.2] for the second one). To estimate the integral on the left vertical side of $Q_{\tau}$, we notice that, for sufficiently large $\tau>0$ and $C_{0}+1 / 2-C_{1} \geq 1$, expansion (2.12), condition (M2) and (3.8) imply that

$$
\begin{aligned}
|\Gamma(s) \tilde{D}(s, w)|= & \left|\sum_{l=1}^{\infty} \frac{(-w)^{l}}{l !} \Gamma(s+l) D(s+l)\right| \\
\leq & \sum_{1 \leq l \leq C_{0}+1 / 2-C_{1}}|\Gamma(s+l) D(s+l)| \frac{w^{l}}{l !} \\
& +\sum_{l>C_{0}+1 / 2-C_{1}}|\Gamma(s+l) D(s+l)| \frac{w^{l}}{l !} \\
= & O\left(e^{-\pi \tau / 2}\right)+O\left(\tau^{-\left\{C_{0}+1 / 2-C_{1}\right\}} e^{-\pi \tau / 2}\left(\sum_{l>C_{0}+1 / 2-C_{1}} \frac{(w \tau)^{l}}{l !}\right)\right) \\
= & O\left(\tau^{-\left\{C_{0}+1 / 2-C_{1}\right\}} e^{-\pi \tau / 2} \sum_{l=1}^{\infty} \frac{(w \tau)^{l}}{l !}\right)=O\left(\tau^{-p} e^{-\tau(\pi / 2-w)}\right),
\end{aligned}
$$


where $p=\left\{C_{0}+1 / 2-C_{1}\right\}$. It is clear that if $C_{0}+1 / 2-C_{1}<1$, the last estimate holds with $p=0$. Combining this observation with (3.7), we obtain

$$
\begin{aligned}
& \left|\frac{1}{2 \pi i} \int_{-C_{0}-i \tau}^{-C_{0}+i \tau} \delta_{n}^{-s} \Gamma(s) \zeta(s+1) \tilde{D}(s, w) d s\right| \\
& =O\left(\delta_{n}^{C_{0}} \int_{-\infty}^{\infty}|y|^{C_{2}-p} e^{-|y|(\pi / 2-w)} d y\right)=O\left(\delta_{n}^{C_{0}}\right) .
\end{aligned}
$$

The last integral converges at 0 and $\pm \infty$ since $C_{2}>0,0 \leq p<1$ and $w<1<\pi / 2$.

Using the uniform convergence of $\Gamma(s) \tilde{D}(s, w)$ whenever $-C_{0} \leq \sigma \leq 1+r$, condition (M2), (3.7) and (3.8), in a similar way we conclude that both integrals over the horizontal segments of the contour $Q_{\tau}$ tend to 0 as $\tau \rightarrow \infty$.

Hence, by (3.2), (3.3), (3.5), (3.6) and (3.9),

$$
H_{n}(w)=J_{n}(w)+O\left(\delta_{n}^{C_{0}}\right), \quad 0 \leq w<1 .
$$

Our proof continues with the application of the residue theorem to the contour integral $J_{n}(w)$. From Lemma 1(ii) and (3.3) it follows that the function $\tilde{D}$ has simple poles at $s=r-1, \ldots, d(r)$ whose residuals can be computed using (2.12). Hence, the integrand in (3.6) has also simple poles at the same points. It is easy to check that the residue at the point $s=r-j, 1 \leq j \leq r-d(r)$, equals $A \Gamma(r) \delta_{n}^{-(r-j)} \zeta(r-j+1)(-w)^{j} / j$ !. Denoting by $V_{n}(r, w)$ the sum of these residuals, we have

$$
V_{n}(r, w)=A \Gamma(r) \sum_{1 \leq j \leq r-d(r)} \delta_{n}^{-(r-j)} \zeta(r-j+1)(-w)^{j} / j !
$$

Next, from the Laurent expansions of the Riemann zeta function $\zeta(s+1)=\frac{1}{s}+\gamma+\ldots$ and the gamma function $\Gamma(s)=\frac{1}{s}-\gamma+\ldots$, where $\gamma$ denotes Euler's constant, and the Taylor expansions of the two remaining factors in (3.6), one concludes that the integrand has also a pole of second order at $s=0$ with residue $\tilde{D}_{s}^{\prime}(0, w)-\tilde{D}(0, w) \log \delta_{n}$. Hence (3.10) implies that

$$
H_{n}(w)=V_{n}(r, w)+\tilde{D}_{s}^{\prime}(0, w)-\tilde{D}(0, w) \log \delta_{n}+O\left(\delta_{n}^{C_{0}}\right), \quad 0 \leq w<1 .
$$

The values $\tilde{D}(0, w)$ and $\tilde{D}_{s}^{\prime}(0, w)$ depend on $r$ and $w$. Their computation stems from the series expansion (2.12) in Lemma 1. It shows that

$$
\tilde{D}(s, w)=\sum_{l=1}^{\infty} \frac{(-w)^{l}}{l !} s(s+1) \ldots(s+l-1) D(s+l) .
$$

If $r$ is not an integer, then the series in the right-hand side of (3.13) represents a function that is analytic at $s=0$ and obviously $\tilde{D}(0, w)=0$. If $r$ is an integer, then condition (M1) implies that $s=0$ is a simple pole of $D(s+r)$ with residue $A$. Writing the Laurent expansion of $D(s+r)$, we get that

$$
\begin{aligned}
\tilde{D}(0, w) & =\left.\frac{(-w)^{r}}{r !} s(s+1) \ldots(s+r-1)\left(\frac{A}{s}+C+\ldots\right)\right|_{s=0} \\
& =\left.\frac{(-w)^{r}}{r !}(s+1) \ldots(s+r-1)(A+C s+\ldots)\right|_{s=0}=\frac{A(-w)^{r}}{r} .
\end{aligned}
$$


We summarize these two observations as follows:

$$
\tilde{D}(0, w)=\left\{\begin{array}{lll}
0 & \text { if } & r \text { is not an integer }, \\
\frac{A(-w)^{r}}{r} & \text { if } & r \text { is an integer. }
\end{array}\right.
$$

To find the value $\tilde{D}_{s}^{\prime}(0, w)$ if $r$ is not an integer, we have to differentiate the right-hand side of (3.13) term by term and set there $s=0$. If $r$ equals an integer, we can proceed in the same way with all terms in (3.13) for which $l \neq r$. If $r=1$, then, by the Laurent expansion $D(s+1)=\frac{A}{s}+C+\ldots$ (see $(1.10)$ ), the first term of the right-hand side of (3.13) becomes

$$
(-w) s\left(\frac{A}{s}+C+\ldots\right)=(-w)(A+C s+\ldots),
$$

whose derivative at $s=0$ equals $C(-w)$. Using the same argument one can easily check that, for $r=$ an integer $>1$, the derivative of the $r$ th term at $s=0$ is equal to $\frac{(-w)^{r}}{r}\left(A h_{r-1}+C\right)$, where $h_{r-1}$ denotes the $(r-1)$ th harmonic number: $h_{r-1}=1+\frac{1}{2}+$ $\ldots+\frac{1}{r-1}$. Defining also $h_{0}=0$, we obtain the following formula:

$$
\tilde{D}_{s}^{\prime}(0, w)= \begin{cases}\sum_{l=1}^{\infty} \frac{(-w)^{l}}{l} D(l) & \text { if } r \text { is not an integer } \\ \sum_{l=1, l \neq r}^{\infty} \frac{(-w)^{l}}{l} D(l)+\frac{(-w)^{r}}{r}\left(A h_{r-1}+C\right) & \text { if } r \text { is an integer. }\end{cases}
$$

We summarize these computations with a formula for $\varphi_{n}\left(e^{-w \delta_{n}}\right)$ on which we base our further asymptotic analysis. We obtain it combining (3.1), (3.12), (3.14) and (3.15). We have

$$
\theta_{n}(r, w) \varphi_{n}\left(e^{-w \delta_{n}}\right)=\exp \left(\tilde{D}_{s}^{\prime}(0, w)+O\left(\delta_{n}^{C_{0}}\right)\right), \quad 0 \leq w<1
$$

where

$$
\theta_{n}(r, w)= \begin{cases}e^{-V_{n}(r, w)} & \text { if } r \text { is not an integer } \\ e^{-V_{n}(r, w)} \delta_{n}^{\frac{A(-w)^{r}}{r}} & \text { if } r \text { is an integer. }\end{cases}
$$

Expressions for $V_{n}(r, w)$ and $\tilde{D}_{s}^{\prime}(0, w)$ are given in (3.11) and (3.15), respectively. Moreover, if $w=w_{n}(t)$, with $w_{n}(t)$ given by (2.29), by virtue of (3.4) we get

$$
\theta_{n}\left(r, w_{n}(t)\right) \varphi_{n}\left(e^{-w_{n}(t) \delta_{n}}\right)=\exp \left(-\lambda_{r}^{2} t^{2} / 2+\tilde{D}_{s}^{\prime}\left(0, w_{n}(t)\right)+O\left(\delta_{n}^{C_{0}}\right)\right) .
$$

\subsection{The Asymptotic Behavior of $\varphi_{n}\left(e^{-w \delta_{n}}\right)$}

Case (i): $0<r<1$. From (2.13) it follows that the sum of residues $V_{n}(r, w)$ in (3.11) is either 0 (if $r-1 \leq-C_{0}$ ) or it contains exactly one summand of order $O\left(\delta_{n}^{1-r}\right)$ (if $\left.r-1>-C_{0}\right)$. Hence, by (3.15)-(3.17),

$$
\left(1+O\left(\delta_{n}^{1-r}\right)\right) \varphi_{n}\left(e^{-w \delta_{n}}\right) \sim \exp \left(\sum_{l=1}^{\infty} \frac{(-w)^{l}}{l} D(l)+O\left(\delta_{n}^{C_{0}}\right)\right) .
$$


On the other hand, expanding $D(l)$ as a Dirichlet series (1.5) and changing the order of summation, we obtain

$$
\begin{aligned}
\exp \left(\sum_{l=1}^{\infty} \frac{(-w)^{l}}{l} D(l)\right) & =e^{-D(1) w} \exp \left(\sum_{l \geq 2} \frac{(-w)^{l}}{l} \sum_{k \geq 1} \frac{b_{k}}{k^{l}}\right) \\
& =e^{-D(1) w} \exp \left(-\sum_{k \geq 1} b_{k}(\log (1+w / k)-w / k)\right) \\
& =e^{D(1)(-w)} \prod_{k=1}^{\infty}\left(1-\frac{(-w)}{k}\right)^{-b_{k}} e^{-b_{k}(-w) / k}
\end{aligned}
$$

In the above computation we have separated the first factor with $D(1)$ from the others in order to get as a limit a function that can be interpreted in terms of the class of functions studied in detail by Hirschman and Widder [10; Chap. III] (see (1.16) and (1.18) and Remark 3 in our Introduction). These observations show that

$$
\lim _{n \rightarrow \infty} \varphi_{n}\left(e^{-w \delta_{n}}\right)=e^{D(1)(-w)} \prod_{k=1}^{\infty}\left(1-\frac{(-w)}{k}\right)^{-b_{k}} e^{-b_{k}(-w) / k}, 0 \leq w<1 .
$$

Case (ii): $r=1$. We have $V_{n}(r, w)=0$ by (2.13). Then (3.15)-(3.17) imply that

$$
\begin{aligned}
\lim _{n \rightarrow \infty} \delta_{n}^{A(-w)} \varphi_{n}\left(e^{-w \delta_{n}}\right) & =\exp \left(C(-w)+\sum_{l=2}^{\infty} \frac{(-w)^{l}}{l} D(l)\right) \\
& =e^{C(-w)} \prod_{k=1}^{\infty}\left(1-\frac{(-w)}{k}\right)^{-b_{k}} e^{-b_{k}(-w) / k}, \quad 0 \leq w<1 .
\end{aligned}
$$

Case (iii): $1<r<2$. It is easily checked, using (2.13), that the right-hand side of (3.11) contains at most two terms. If $\{r\}-1>-C_{0}$, then the corresponding term is of order $O\left(\delta_{n}^{2-r}\right)$. So, in this case we have

$$
V_{n}(r, w)=A \Gamma(r) \zeta(r) \delta_{n}^{-(r-1)}(-w)+O\left(\delta_{n}^{2-r}\right)
$$

Using again (3.15)-(3.17), we obtain the limit from case (i):

$$
\begin{aligned}
& \lim _{n \rightarrow \infty} \exp \left(-A \Gamma(r) \zeta(r) \delta_{n}^{-(r-1)}(-w)\right) \varphi_{n}\left(e^{-w \delta_{n}}\right) \\
& =e^{D(1)(-w)} \prod_{k=1}^{\infty}\left(1-\frac{(-w)}{k}\right)^{-b_{k}} e^{-b_{k}(-w) / k}, \quad 0 \leq w<1 .
\end{aligned}
$$

We conclude these three cases with the observation, that the limits which we have obtained there, are always from the class of functions $1 / K(t)$ discussed in Remark 3 of the Introduction. (For more details, see [10; Chap. III].)

In the next two cases we are interested in w's which depend on $n$ and approach 0 as $n \rightarrow \infty$ (see conditions (iii) and (iv) of Lemma 4). 
Case (iv): $r=2$. From (3.11) we get $V_{n}\left(2, w_{n}(t)\right)=A \delta_{n}^{-1} \zeta(2)\left(-w_{n}(t)\right)$, which by (3.17) in turn yields

$$
\theta_{n}\left(2, w_{n}(t)\right)=\delta_{n}^{A w_{n}^{2}(t) / 2} \exp \left(-A \delta_{n}^{-1} \zeta(2)\left(-w_{n}(t)\right)\right)
$$

Hence, combining (3.15) and (3.16), we conclude that

$$
\begin{aligned}
& \exp \left(-A \delta_{n}^{-1} \zeta(2)\left(-w_{n}(t)\right)\right) \varphi_{n}\left(e^{-w_{n}(t) \delta_{n}}\right) \\
& =\exp \left(\frac{w_{n}^{2}(t)}{2}\left(A+C-A \log \delta_{n}\right)+\sum_{l \geq 1, l \neq 2} \frac{\left(-w_{n}(t)\right)^{l}}{l} D(l)+O\left(\delta_{n}^{C_{0}}\right)\right) .
\end{aligned}
$$

Case $(v): r>2$. This case is studied in a similar way as previously. Using (3.11), (3.15)-(3.18), we obtain

$$
\begin{aligned}
& \exp \left(-A \Gamma(r) \zeta(r) \delta_{n}^{-(r-1)}\left(-w_{n}(t)\right)\right) \varphi_{n}\left(e^{-w_{n}(t) \delta_{n}}\right) \\
& =\exp \left(A \Gamma(r) \zeta(r-1) \delta_{n}^{-(r-2)} \frac{w_{n}^{2}(t)}{2}+\sum_{l \geq 1} \frac{\left(-w_{n}(t)\right)^{l}}{l} D(l)+R_{n}\left(r, w_{n}(t)\right)\right) \\
& \times \exp \left(-\lambda_{r}^{2} t^{2} / 2\right),
\end{aligned}
$$

where

$$
\begin{aligned}
R_{n}(r, w) & =A \Gamma(r) \sum_{2<j \leq r-d(r)} \delta_{n}^{-(r-j)} \zeta(r-j+1)\left(-w_{n}(t)\right)^{j} / j ! \\
& + \begin{cases}\frac{(-w)^{r}}{r}\left(A h_{r-1}+C-A \log \delta_{n}\right)+O\left(\delta_{n}^{C_{0}}\right) & \text { if } r \text { is an integer, } \\
O\left(\delta_{n}^{C_{0}}\right) & \text { if } r \text { is not an integer. }\end{cases}
\end{aligned}
$$

We assume that the sum in the right-hand side of (3.25) is $=0$ if $r-d(r) \leq 2$.

\subsection{End of the Proof: The Application of a Continuity Theorem}

Consider first the values of the parameter $r$ given in parts (i)-(iii) of our theorem and recall the limits established in (3.20)-(3.22) of the previous subsection. It is immediately seen that if we change the variable $w$ by $t=-w$ in (3.20)-(3.22), then their right-hand sides become the functions $g_{1}$ and $g_{2}$ representing formally the moment generating function $E\left(e^{t \xi}\right),-1<t \leq 0$, in (1.8) and (1.9) of parts (i)-(iii) of our theorem, respectively. Moreover, the substitution $u=e^{t \delta_{n}}$ in the probability generating functions $\varphi_{n}(u$ ) (see also (1.2)) implies that the left-hand sides of (3.20)-(3.22) are the moment generating functions of the sequences $\eta_{n}^{(1)}=\xi_{n} \delta_{n}$ (case (i)), $\eta_{n}^{(2)}=\xi_{n} \delta_{n}+A \log \delta_{n}$ (case (ii)) and $\eta_{n}^{(3)}=\xi_{n} \delta_{n}-A \Gamma(r) \zeta(r) \delta_{n}^{-(r-1)}, n \geq 1$ (case (iii)), respectively. Eqs. (3.20)-(3.22) hold uniformly for $w \in[0,1)$. Hence they are valid for any $t \in(-b,-a) \subset(-1,0)$ and the corresponding sequences of moment generating functions converge to the moment generating functions $g_{1}$ or $g_{2}$ of the random variable $\xi$ defined in parts (i)-(iii) of the 
theorem. Therefore, we can apply the continuity theorem [17; Thm. 2] and establish that the sequences of the distribution functions of the above random variables converge to the distribution functions of the random variable $\xi$ defined in parts (i)-(iii) of the theorem. (In fact, Thm. 2 in [17] requires that $0<a<t<b$, so that it implies the weak convergence of $-\eta_{n}^{(j)}, j=1,2,3$, to the random variable $-\xi$, which is obviously equivalent to our statement.) Let $G_{j}(x)$ denote the distribution functions whose moment generating functions are $g_{j}(t), j=1,2$, respectively. The continuity theorem [17; Thm.2] implies that at each continuity point $x$ of the functions $G_{j}(x)$ we have

$$
\begin{aligned}
& G_{n, 1}(x)=P\left(\eta_{n}^{(1)} \leq x\right) \rightarrow G_{1}(x) \quad(\text { case }(\mathrm{i})) \\
& G_{n, 2}(x)=P\left(\eta_{n}^{(2)} \leq x\right) \rightarrow G_{2}(x) \quad(\text { case }(\mathrm{ii})) \\
& G_{n, 3}(x)=P\left(\eta_{n}^{(3)} \leq x\right) \rightarrow G_{1}(x) \quad(\text { case }(\text { iii }))
\end{aligned}
$$

To complete the proof of parts (i)-(iii) of the theorem it remains to show that the convergence in (3.26)-(3.28) holds if the normalizing factor $\delta_{n}$ is replaced by $1 / L_{n}(r)$ (see $(1.7)$ ). To show this we first set

$$
\begin{gathered}
Y_{n, 1}=\xi_{n} / L_{n}(r) \quad(\text { case }(\mathrm{i})) \\
Y_{n, 2}=\xi_{n} / L_{n}(1)-A \log L_{n}(1) \quad(\text { case (ii)), } \\
Y_{n, 3}=\xi_{n} / L_{n}(r)-A \Gamma(r) \zeta(r) L_{n}^{r-1}(r) \quad(\text { case (iii)). }
\end{gathered}
$$

Using (2.8) and (1.7), after standard algebraic manipulations, for each fixed $n \geq 1$, we obtain

$$
Y_{n, j}=q_{n, j} \eta_{n}^{(j)}+\Delta_{n, j}, \quad j=1,2,3
$$

where

$$
\begin{gathered}
q_{n, 1}=1 / \delta_{n} L_{n}(r), \quad \Delta_{n, 1}=0 \\
q_{n, 2}=\frac{1}{\delta_{n} L_{n}(1)}, \quad \Delta_{n, 2}=-\frac{A \log \delta_{n}}{\delta_{n} L_{n}(1)}-A \log L_{n}(1), \\
q_{n, 3}=\frac{1}{\delta_{n} L_{n}(r)}, \quad \Delta_{n, 3}=\frac{A \Gamma(r) \zeta(r) \delta_{n}^{-(r-1)}}{\delta_{n} L_{n}(r)}-A \Gamma(r) \zeta(r) L_{n}^{r-1}(r) .
\end{gathered}
$$

These relations imply that $q_{n, j}=1+O\left(n^{-\frac{r}{r+1}}\right), j=1,2,3$, and $\Delta_{n, 1}=0, \Delta_{n, 2}=$ $O\left(n^{-1 / 2} \log n\right), \Delta_{n, 3}=O\left(n^{-\frac{1}{r+1}}\right)$, as $n \rightarrow \infty$. Generally speaking, we observe the relationship

$$
Y_{n}=q_{n} \eta_{n}+\Delta_{n}
$$

where $q_{n}, n \geq 1$, and $\Delta_{n}, n \geq 1$, are sequences of reals such that $\lim _{n \rightarrow \infty} q_{n}=1$ and $\lim _{n \rightarrow \infty} \Delta_{n}=0$, while the sequence $\eta_{n}, n \geq 1$, of random variables converges weakly to the random variable $\xi$ as $n \rightarrow \infty$ (we drop the second subscripts for the sake of convenience). We have to show that the sequence $Y_{n}, n \geq 1$, defined by (3.29), converges weakly to $\xi$ as well. We shall apply a standard probabilistic argument. We first set $q_{n}=1+\epsilon_{n}, n \geq 1$, $\lim _{n \rightarrow \infty} \epsilon_{n}=0$ and assume that $x$ is a point of continuity of the distribution function $G(x)=P(\xi \leq x)$. (Note that Hirschman and Widder [10; Chap. III, Sect. 6] have proved 
that the distribution functions corresponding to the limiting moment generating functions $g_{1}$ and $g_{2}$ in cases (i)-(iii) are everywhere continuous.) Then, from (3.29) it follows that

$$
\begin{aligned}
& F_{n}(x):=P\left(Y_{n} \leq x\right)=P\left(\eta_{n} \leq \frac{x-\Delta_{n}}{1+\epsilon_{n}}\right) \\
& =P\left(\eta_{n} \leq x-\frac{x \epsilon_{n}+\Delta_{n}}{1+\epsilon_{n}}\right)=G_{n}\left(x-\frac{x \epsilon_{n}+\Delta_{n}}{1+\epsilon_{n}}\right), n \geq 1
\end{aligned}
$$

Taking now an arbitrary $\epsilon>0$ and $n$ enough large so that $-\epsilon<\left(x \epsilon_{n}+\Delta_{n}\right) /\left(1+\epsilon_{n}\right)<\epsilon$, for fixed $x$, we obtain

$$
G_{n}(x-\epsilon) \leq F_{n}(x) \leq G_{n}(x+\epsilon) .
$$

Letting $n \rightarrow \infty$ in the above inequalities, we find that

$$
G(x-\epsilon) \leq \lim \inf _{n \rightarrow \infty} F_{n}(x) \leq \lim \sup _{n \rightarrow \infty} F_{n}(x) \leq G(x+\epsilon)
$$

for all $\epsilon>0$. Since $G$ is continuous at $x$ then

$$
G(x-\epsilon) \rightarrow G(x) \quad \text { and } \quad G(x+\epsilon) \rightarrow G(x)
$$

as $\epsilon \rightarrow 0^{+}$and the result is proved. With this the proof of cases (i)-(iii) is completed.

We proceed to the proofs of the remaining two parts of our theorem. In each case $w_{n}$ will be suitably chosen in order to get the required weak convergence to the Gaussian distribution. Our argument stems from (3.23)-(3.25). To prove part (iv) we set in (3.23)

$$
w_{n}(t)=-\frac{t}{\sqrt{A\left(-\log \delta_{n}\right)}}, \quad t \in(a, b) \subset(-1,0) .
$$

Then its left-hand side becomes

$$
\begin{aligned}
& \exp \left(-\frac{A \delta_{n}^{-1} \zeta(2) t}{\sqrt{-A \log \delta_{n}}}\right) \varphi_{n}\left(\exp \left(\frac{t \delta_{n}}{\sqrt{-A \log \delta_{n}}}\right)\right) \\
& =E\left(\exp \left(t \frac{\xi_{n} \delta_{n}-A \delta_{n}^{-1} \zeta(2)}{\sqrt{-A \log \delta_{n}}}\right)\right)=E\left(\exp \left(t \frac{\xi_{n}-A \delta_{n}^{-2} \zeta(2)}{\delta_{n}^{-1} \sqrt{-A \log \delta_{n}}}\right)\right) .
\end{aligned}
$$

On the other hand, a passage to the limit, as $n \rightarrow \infty$, in the right-hand side of (3.23) and the substitution (3.30) imply that

$$
\lim _{n \rightarrow \infty} E\left(\exp \left(t \frac{\xi_{n}-A \delta_{n}^{-2} \zeta(2)}{\delta_{n}^{-1} \sqrt{-A \log \delta_{n}}}\right)\right)=e^{t^{2} / 2} .
$$

Applying in the same manner as in the previous cases the continuity theorem for moment generating functions [17; Thm. 2], we conclude that the sequence of the distribution functions of the random variables $\eta_{n}=\left(\xi_{n}-A \delta_{n}^{-2} \zeta(2)\right) / \delta_{n}^{-1} \sqrt{-A \log \delta_{n}}$ converges weakly to the distribution function of the standard Gaussian distribution. Now, from (2.8) and (1.7) it follows that

$$
\delta_{n}^{-1}=L_{n}(2)\left(1+O\left(n^{-2 / 3}\right)\right)
$$


and therefore,

$$
-\log \delta_{n}=\left(-\log \left(\left(\frac{n}{2 A \zeta(3)}\right)^{-1 / 3}\right)\right)\left(1+O\left(n^{-2 / 3}\right)\right)=\frac{\log n}{3}+O(1) .
$$

The proof of part (iv) is now completed in the same way as in parts (i)-(iii), setting $Y_{n}=\left(\xi_{n}-\mu_{n}\right) / \sigma_{n}\left(\mu_{n}\right.$ and $\sigma_{n}$ are given by (1.12)) and using again (3.29) with $q_{n}=$ $1+O(1 / \sqrt{\log n}), \Delta_{n}=O\left(n^{-1 / 3} / \sqrt{\log n}\right)$. Hence we obtain the convergence given by (1.11).

Finally, in part (v) we set

$$
\begin{aligned}
& w_{n}(t)=-\frac{t}{\left(A \Gamma(r) \zeta(r-1) \delta_{n}^{-(r-2)}\right)^{1 / 2}}=-\frac{t \delta_{n}^{r / 2-1}}{(A \Gamma(r) \zeta(r-1))^{1 / 2}}, \\
& t \in(-b,-a) \subset(-1,0),
\end{aligned}
$$

and establish in a similar way, using (3.4), (3.24) and (3.25), that

$$
\begin{aligned}
& \lim _{n \rightarrow \infty} \exp \left(-\frac{A \Gamma(r) \zeta(r) \delta_{n}^{-(r-1)} t}{\left(A \Gamma(r) \zeta(r-1) \delta_{n}^{-(r-2)}\right)^{1 / 2}}\right) \\
& \times \varphi_{n}\left(\exp \left(\frac{t \delta_{n}}{\left(A \Gamma(r) \zeta(r-1) \delta_{n}^{-(r-2)}\right)^{1 / 2}}\right)\right) \\
& =\lim _{n \rightarrow \infty} E\left(\exp \left(t \frac{\xi_{n} \delta_{n}-A \Gamma(r) \zeta(r) \delta_{n}^{-(r-1)}}{\left(A \Gamma(r) \zeta(r-1) \delta_{n}^{-(r-2)}\right)^{1 / 2}}\right)\right) \\
& =\lim _{n \rightarrow \infty} E\left(\exp \left(t \frac{\xi_{n}-A \Gamma(r) \zeta(r) \delta_{n}^{-r}}{\left(A \Gamma(r) \zeta(r-1) \delta_{n}^{-r}\right)^{1 / 2}}\right)\right)=e^{\left(1-\lambda_{r}^{2}\right) t^{2} / 2}
\end{aligned}
$$

where $\lambda_{r}$ is defined by (2.30). The last exponent is the moment generating function of a Gaussian distribution with zero mean and variance $1-\lambda_{r}^{2}$. Hence, by the continuity theorem [17; Thm. 2] we obtain in the same way that the random variable

$$
\eta_{n}^{\prime}=\frac{\xi_{n}-A \Gamma(r) \zeta(r) \delta_{n}^{-r}}{\left(A \Gamma(r) \zeta(r-1) \delta_{n}^{-r}\right)^{1 / 2}}
$$

converges weakly to the same Gaussian distribution, or equivalently, by (3.31),

$$
\eta_{n}=\frac{\eta_{n}^{\prime}}{\sqrt{1-\lambda_{r}^{2}}}=\frac{\xi_{n}-A \Gamma(r) \zeta(r) \delta_{n}^{-r}}{\left(A \Gamma(r)\left(\zeta(r-1)-\frac{(r-1)^{2} \zeta^{2}(r)}{r(r+1) \zeta(r+1)}\right) \delta_{n}^{-r}\right)^{1 / 2}}
$$

converges weakly to the standard Gaussian distribution. If we set $Y_{n}=\left(\xi_{n}-\mu_{n}^{\prime}\right) / \sigma_{n}^{\prime}$, where $\mu_{n}^{\prime}$ and $\sigma_{n}^{\prime}$ are given by (1.14), then it is not difficult to show that (3.29) holds with $q_{n}=1+O\left(n^{-\frac{r}{r+1}}\right)$ and $\Delta_{n}=O\left(n^{-\frac{r}{r+1}}\right)$, which in turn implies the convergence in (1.13). 


\section{Examples}

4.1. Integer partitions. Brief historical remarks on the number of parts in linear integer partitions were given in the Introduction. We recall (see e.g. [2; Chap. 1]) that such partitions are defined as the $m$-tuple $\omega=\left(\omega_{1}, \omega_{2}, \ldots, \omega_{m}\right)$ such that $n=\omega_{1}+\omega_{2}+\ldots+\omega_{m}$, where the positive integers $\omega_{j}$ satisfy $\omega_{1} \geq \omega_{2} \geq \ldots \geq \omega_{m}$. The total number of such partitions (in our notation $c_{n}$ ) is determined asymptotically, as $n \rightarrow \infty$, by the partition formula of Hardy and Ramanaujan [8]. In [2; Chap. 6] this formula is also derived as a consequence of Meinardus' theorem [16]. The generating function of the sequence $c_{m, n}, 1 \leq m \leq n, n \geq 1$, satisfies (1.1) with $b_{k}=1, k \geq 1$ (see [25; Sect. 3.14] or [2; Chap. $2]$ ). Therefore, $D(s)=\zeta(s), D(s, w)=\zeta(s, 1+w)$ (see (2.14)) and, by known properties of the Hurwitz zeta function [24; Sect. 13.13], we have to set $A=r=1, C=\gamma$ in (1.9), where $\gamma$ denotes Euler's constant. Moreover, by (3.3) we have $\tilde{D}(s, w)=\zeta(s, 1+w)-\zeta(s)$. Hence, from the known formulas $\frac{d}{d s} \zeta(s, 1+w)=\log \Gamma(1+w)-\log \sqrt{2 \pi}$ and $\zeta^{\prime}(0)=-\log \sqrt{2 \pi}[24$; Sect.13.21] it follows that $\tilde{D}_{s}^{\prime}(0, w)=\log \Gamma(1+w)$. On the other hand, from $(2.13),(3.11)$ and (3.17) it follows that $V_{n}(1, w)=0$ and $\theta_{n}(1, w)=\delta_{n}^{-A w}$. Therefore, (3.16) implies that $\lim _{n \rightarrow \infty} \delta_{n}^{A t} \varphi_{n}\left(e^{t \delta_{n}}\right)=\lim _{n \rightarrow \infty} E\left(\exp \left(t\left(\xi_{n} \delta_{n}+A \log \delta_{n}\right)=\Gamma(1-t), t \in(a, b) \subset(-1,0)\right.\right.$. Hence, the argument given in Subsection 3.3 shows that the limiting moment generating function of the random variable $\xi_{n} / L_{n}(1)-A \log L_{n}(1)=\pi \xi_{n} /(6 n)^{1 / 2}-\log \left((6 n)^{1 / 2} / \pi\right)$ is $\Gamma(1-t)$. The latter one represents the moment generating function of the doubly exponential distribution function $e^{-e^{-x}},-\infty<x<\infty$. This gives the result of Erdös and Lehner [6].

4.2. Plane partitions. A plane partition $\omega$ of the positive integer $n$ is a representation $n=\sum_{k, l \geq 1} \omega_{k, l}$, in which the array $\left(\omega_{k, l}\right)_{k, l \geq 1}$ of non-negative entries is such that $\omega_{k, l} \geq$ $\omega_{k+1, l}$ and $\omega_{k, l} \geq \omega_{k, l+1}$. The asymptotic of the total number $c_{n}$ of plane partitions of $n$, as $n \rightarrow \infty$, was determined by Wright [26]. In the case of plane partitions $b_{k}=k, k \geq 1[2$; Chap. 11], and thus $D(s)=\zeta(s-1), D(s, w)=\zeta(s-1,1+w)-w \zeta(s, 1+w), r=2$ and $A=1$. Thus, applying the result of Theorem 1 (iv), (2.8) and (1.7), we get the following limiting distribution of the trace $\xi_{n}$ of a random plane partition of $n$ :

$$
\lim _{n \rightarrow \infty} P\left(\frac{\xi_{n}-\zeta(2)(n / 2 \zeta(3))^{2 / 3}}{(n / 2 \zeta(3))^{1 / 3}\left(\frac{1}{3} \log n\right)^{1 / 2}} \leq x\right)=\frac{1}{\sqrt{2 \pi}} \int_{-\infty}^{x} e^{-y^{2} / 2} d y,-\infty<x<\infty .
$$

This result is obtained in [12] using a different method.

4.3. Generalized Bose-Einstein model of ideal gas. In the Introduction we pointed out that weighted integer partitions are associated with the $d$-dimensional model of quantum ideal gas, where $n$ is interpreted there as the total energy of the system of particles. The weights $b_{k}, k \geq 1$, are viewed as counts of the distinct positions of the particles in the phase space, where a particle in a given position has (rescaled) energy $k$ (for more details, see $[22,23]$ and the references therein).

Except for the case $b_{k}=\rho k^{r-1}, k \geq 1, \rho, r>0$, where $D(s)=\rho \zeta(s-r+1)$, Vershik and Yakubovich [23] studied a more realistic model in which the weights $b_{j}$ satisfy $\sum_{j=1}^{k} b_{j}=c_{d} k^{d / 2}+E_{d}(k)$ with $c_{d}=\pi^{d / 2} / \Gamma(d / 2+1)$, where $d$ denotes the space dimension. The error terms $E_{d}(k)$ are estimated for large $k$ 's by $E_{d}(k)=O\left(k^{\alpha_{d}}\right), d=1,2, \ldots$, 
where the constants $\alpha_{d}<d / 2$ are explicitly given in [23]. We believe that more accurate estimates for $E_{d}(k)$ would imply expressions for the generating series $D(s)$ in terms of linear combinations of values of the Riemann zeta function which will depend on the dimension $d$. A precise evaluation of the parameters $r=r(d)$ and $A=A(d)$ will then allow a subsequent application of Theorem 1. In this way, one can obtain similar limiting distributions whenever the total energy of the system approaches $\infty$.

\section{Acknowledgements}

The author is thankful to Boris Granovsky for many helpful discussions on the application of Khintchine-Meinardus method. He also wishes to express his gratitude to the referee whose valuable and constructive comments helped eliminate defects in the earlier versions of this paper.

\section{References}

[1] M. Abramovitz and I.A. Stegun, Handbook of Mathemathical Functions with Formulas, Graphs and Mathematical Tables, Dover Publ., Inc (New York, 1965).

[2] G.E. Andrews, The Theory of Partitions, Encyclopedia Math. Appl. 2, AddisonWesley (Reading, MA, 1976).

[3] R. Arratia, A.D. Barbour and S. Tavaré, Logarithmic Combinatorial Structures: a Probabilistic Approach, Europ. Math. Soc. (Zürich, 2003).

[4] F.C. Auluck, S. Chowla and H. Gupta, On the maximum value of the number of partitions of $n$ into $k$ parts, J. Indian Math. Soc., 6 (1942), 105-112.

[5] J. Curtiss, A note on the theory of moment generating functions, Ann. Math. Statist., 13 (1942), 430-433.

[6] P. Erdős and J. Lehner, The distribution of the number of summands in the partition of a positive integer, Duke. Math.J., 8 (1941), 335-345.

[7] B. Granovsky, D. Stark and M. Erlihson, Meinardus theorem on weighted partitions: Extensions and a probabilistic proof, Adv. Appl. Math., 41 (2008), 307-328.

[8] G.H. Hardy and S. Ramanujan, Asymptotic formulae in combinatory analysis, Proc. London Math. Soc., 17 (1918), 75-115.

[9] C.B. Haselgrove and H.N.V. Temperley, Asymptotic formulae in the theory of partitions, Proc. Cambr. Phil. Soc., 50 (1954), 225-241.

[10] J.J Hirschman and D.V. Widder, The Convolution Transform, Princeton Univ. Press (Princeton, 1955).

[11] H.-K. Hwang, Limit theorems for the number of summands in integer partitions, $J$. Combinatorial Theory, Ser. A, 96 (2001), 89-126.

[12] E. Kamenov and L. Mutafchiev, The limiting distribution of the trace of a random plane partition, Acta Math. Hung., 117 (2007), 293-314. 
[13] A.I. Khinchin, Mathematical Foundations of Quantum Statistics, Graylock Press (Albany, NY, 1960).

[14] D.V. Lee, The asymptotic distribution of the number of summands in unrestricted $\Lambda$-partitions, Acta Arith., 65 (1993), 29-43.

[15] E. Lukacs, Characteristic Functions, 2nd ed., Griffin (London, 1970).

[16] G. Meinardus, Asymptotische Aussagen über Partitionen, Math. Z., 59 (1954), 388398.

[17] A. Mukherjea, M. Rao and S. Suen, A note on moment generating functions, Statist. Probab. Letters, 76 (2006), 1185-1189.

[18] A. Nijenhuis and H. Wilf, Combinatorial Algorithms, 2nd Ed., Academic Press, (New York, 1978).

[19] L.B. Richmond, Some general problems on the number of parts in partitions, Acta Arith., 66 (1994), 297-314.

[20] R.P. Stanley, Enumerative Combinatorics 2, Vol. 62 of Cambridge Studies in Advanced Mathematics, Cambridge Univ. Press (Cambridge, 1999).

[21] E.C. Titchmarsh, The Theory of Functions, Oxford Univ. Press (Oxford, 1939).

[22] A. Vershik, Statistical mechanics of combinatorial partitions and their limit configurations, Funct. Anal. Appl., 30 (1996), 90-105.

[23] A. Vershik and Yu. Yakubovich, Fluctuations of the maximal particle energy of the quantum ideal gas and random partitions, Comm. Math. Phys., 261 (2006), 759-769.

[24] E.T. Whittaker and G.N. Watson, A Course of Modern Analysis, Cambridge Univ. Press (Cambridge, 1927).

[25] H. Wilf, generatingfunctionology, Academic Press (New York, 1994).

[26] E.M. Wright, Asymptotic partition formulae, I: Plane partitions, Quart. J. Math. Oxford Ser.(2), 2 (1931), 177-189. 